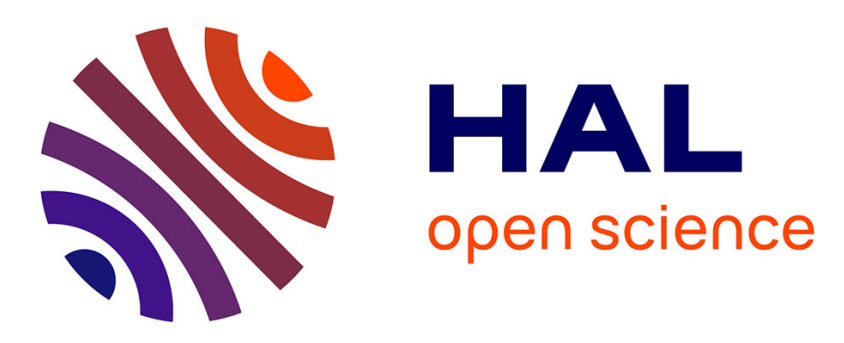

\title{
Adaptive IEEE 802.15.4 MAC for Throughput and Energy Optimization
}

Nazim Abdeddaim, Fabrice Theoleyre, Martin Heusse, Andrzej Duda

\section{To cite this version:}

Nazim Abdeddaim, Fabrice Theoleyre, Martin Heusse, Andrzej Duda. Adaptive IEEE 802.15.4 MAC for Throughput and Energy Optimization. DCOSS 2013 - IEEE International Conference on Distributed Computing in Sensor Systems, 2013, Cambridge, Massachusetts, United States. pp.223-230, 10.1109/DCOSS.2013.44 . hal-01073742

\section{HAL Id: hal-01073742 \\ https://hal.inria.fr/hal-01073742}

Submitted on 2 Jun 2020

HAL is a multi-disciplinary open access archive for the deposit and dissemination of scientific research documents, whether they are published or not. The documents may come from teaching and research institutions in France or abroad, or from public or private research centers.
L'archive ouverte pluridisciplinaire HAL, est destinée au dépôt et à la diffusion de documents scientifiques de niveau recherche, publiés ou non, émanant des établissements d'enseignement et de recherche français ou étrangers, des laboratoires publics ou privés. 


\title{
Adaptive IEEE 802.15.4 MAC for Throughput and Energy Optimization
}

\author{
Nazim Abdeddaim*, Fabrice Theoleyre ${ }^{\Uparrow}$, Martin Heusse*, and Andrzej Duda* \\ ${ }^{*}$ Grenoble Institute of Technology, CNRS Grenoble Informatics Laboratory UMR 5217, France \\ ${ }^{\top}$ CNRS, ICUBE, University of Strasbourg, UMR 7005, France \\ Email: \{firstname.lastname\}@imag.fr, theoleyre@unistra.fr
}

\begin{abstract}
IEEE 802.15.4 defines a popular MAC standard for wireless sensor and actuator networks. With the default parameters, under medium to high load, 802.15.4 generates excessive collisions and packet losses. Low duty cycles even exacerbate the problem, because more nodes become active after long periods of sleep and contend for channel access.

In this paper, we have applied the models that led to the Idle Sense access method for 802.11 to the 802.15.4 slotted CSMA/CA, taking into account the central role of the coordinator and also the bursty nature of the traffic. Surprisingly, the approach perfectly applies to 802.15.4 even if the principles of the two access methods fundamentally differ. Based on the model, we propose ABE, an adaptation method that adjusts the contention window to optimal values so that the network obtains high throughput along with low duty cycles leading to low energy consumption. The method converges to near-optimal backoff values even under bursty traffic and for any number of contending nodes.
\end{abstract}

Keywords-IEEE 802.15.4; access method; adaptation; low duty cycle; optimal throughput.

\section{INTRODUCTION}

IEEE 802.15.4 [1] defines a standard for networks of lowpower sensors and actuators with two operating modes. The first one is the non-beacon enabled mode in which receivers need to be awake to receive a frame at any time. This mode does not enable low energy consumption in multihop topologies. The second mode, beacon-enabled, defines superframes that start with the transmission of beacons sent by coordinators. Nodes associated with a coordinator contend for channel access according to a slotted CSMA/CA scheme during the active period at the beginning of the superframe. Long lifetimes are possible with low duty cycles-nodes are only awake during a small part of a superframe and they sleep most of the time [2].

When IEEE 802.15.4 operates with the default parameters defined by the standard in the beacon-enabled mode, any significant traffic causes frequent collisions and packet losses [3], which further leads to increased energy consumption and low throughput. Low duty cycles even exacerbate the problem, because more nodes become active after long periods of sleep and contend for channel access. So, operating IEEE 802.15.4 with low duty cycles requires a mechanism for adapting the MAC parameters to obtain low energy consumption and efficient operation during active periods at the same time. Optimizing the throughput becomes important in 802.15.4 networks with low duty cycles, because the nominal bit rate of $250 \mathrm{~kb} / \mathrm{s}$ is proportionally reduced to the duration of the active period with respect to the superframe size and is shared by all active nodes associated with the same coordinator.

In this paper, we analyze the behavior of IEEE 802.15.4 in the beacon-enabled mode to identify the main performance bottlenecks and apply the optimization approach of the 802.11 Idle Sense [4]. Surprisingly, the approach perfectly fits IEEE 802.15.4 even if the principles of the two access methods essentially differ. However, a simple application of the Idle Sense optimization approach is insufficient, because bursts of traffic that may arise at the beginning of the active period require special handling. Moreover, the contrast between the behaviors of the coordinator and the nodes that turn on their radios as rarely as possible leads to a specific contention control protocol.

We validate the model needed for optimization with two simulators. Based on the model, we design ABE, an adaptation method in which a coordinator observes the number of idle slots before a transmission, adapt the contention parameter $B E$ to the optimal value, and disseminates its value to associated nodes in a beacon. Finally, we evaluate the adaptation method with simulations of different topologies (number of nodes) and under variable traffic that show a significant improvement of throughput, packet delivery ratio, and delay over the standard IEEE 802.15.4 with constant parameters.

\section{IEEE 802.15.4 BACKGROUND}

We provide below a minimal description of the IEEE 802.15.4 beacon-enabled mode. In this mode, a coordinator periodically sends a beacon frame each Beacon Interval $\left(B I=\right.$ aBaseSuperFrameDuration $\left.* 2^{B O}\right)$ to start a superframe. Nodes associated with the coordinator use the slotted CSMA/CA method to contend for a transmission during the Contention Access Period (CAP) and they may also reserve a Guaranteed TimeSlot (GTS) for periodic real time traffic. The active part of the superframe lasts for a Superframe Duration $\left(S D=\right.$ aBaseSuperFrameDuration $\left.* 2^{S O}\right)$ and nodes may go to sleep after a transmission until the next beacon. The coordinator should stay awake during at least the active period.

The slotted CSMA/CA method consists of locating the next backoff boundary and choosing a random backoff in the contention window between 0 and $\left(2^{B E}-1\right) *$ aUnitBackoffPeriod. A node go to sleep during the backoff and then wakes up to perform two Clear Channel Assessments (CCA) to check if there is an ongoing transmission on the channel. If the channel is sensed busy during a CCA, the node performs an exponential backoff-it chooses a new backoff in an increased 


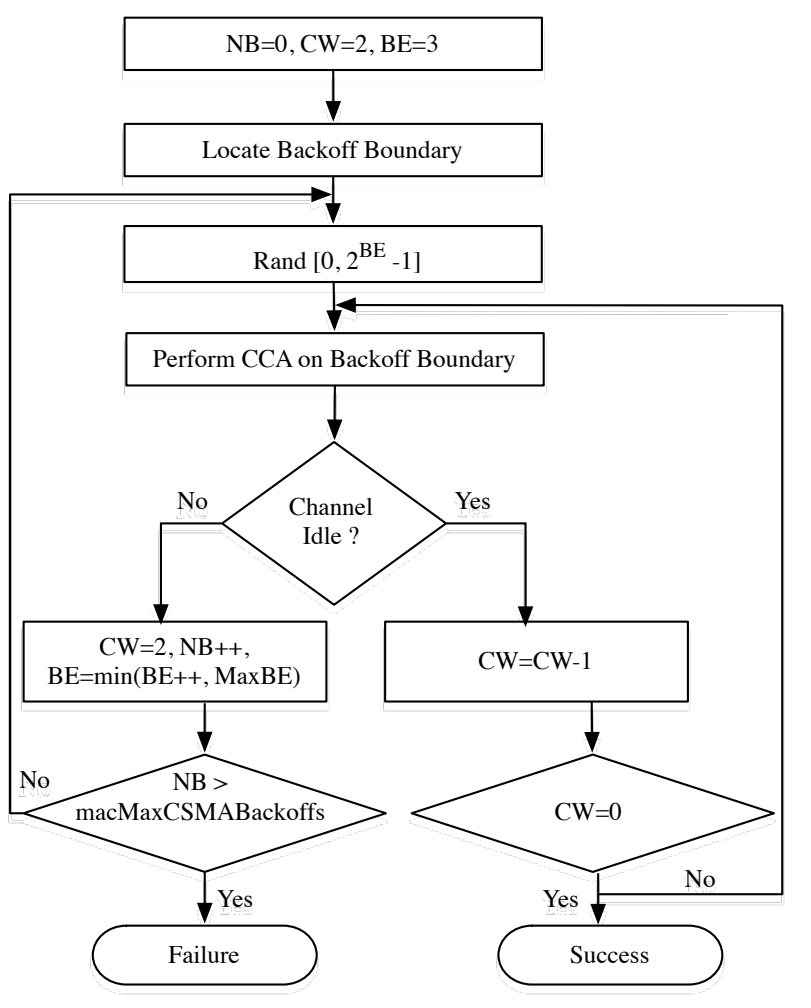

Fig. 1. IEEE 802.15.4 Slotted CSMA/CA

interval between 0 and $\left(2^{B E+1}-1\right) *$ aUnitBackoffPeriod. A node drops a frame either when there is no ACK received after macMaxFrameRetries or when the node performed macMaxCSMABackoffs without finding the channel free.

\section{ChOICE OF MAC PARAMETERS}

Low energy consumption is vital in Wireless Sensor Networks and nodes can achieve very low duty cycles (the proportion between awake and sleep periods) by extending the sleeping periods or reducing active periods. For instance, with the beacon parameters $S O=B O-10$, the duty cycle is as low as $0.1 \%\left(2^{-10}\right)$ for the coordinator (it needs to stay awake during the active period) and an order of magnitude lower for the devices that only need to wake up for the beacon reception and possibly a CCA, and frame transmission.

We have first studied the impact of different MAC parameters on the IEEE 802.15.4 performance in a simple simulation set up:

- macMaxFrameRetries: number of retransmissions (because no ACK received) before dropping a frame,

- macMaxCSMABackoffs: number of unsuccessful channel sensing before dropping a frame,

- $B E$ : backoff exponent that determines the size of the contention window from which a node chooses the value of the random backoff interval before sensing the channel.

We have used WsNet, an event-driven simulator for large scale wireless sensor networks [5] and an open source implementation of IEEE 802.15.4 for WsNet [6] (it implements the Beacon-Only Period [7] and beacon-enabled mode). We

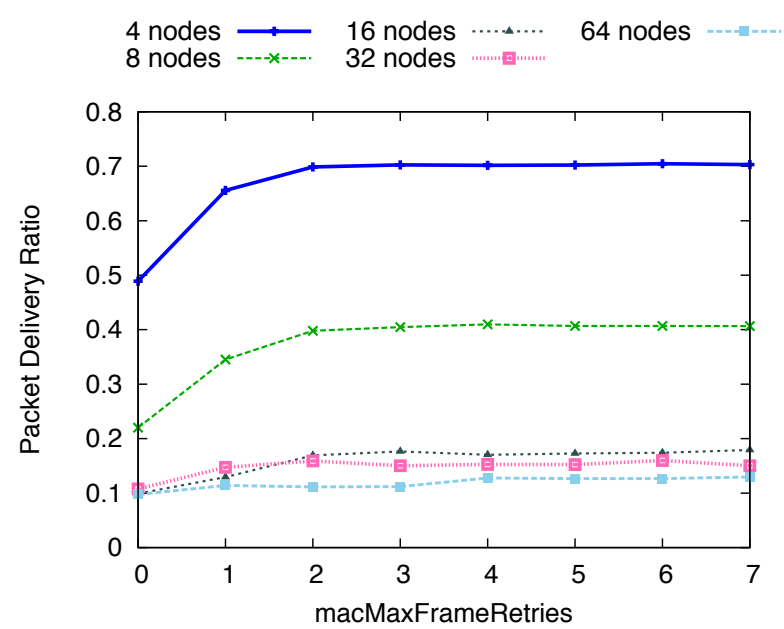

(a) Packet Delivery Ratio in function of macMaxFrameRetries

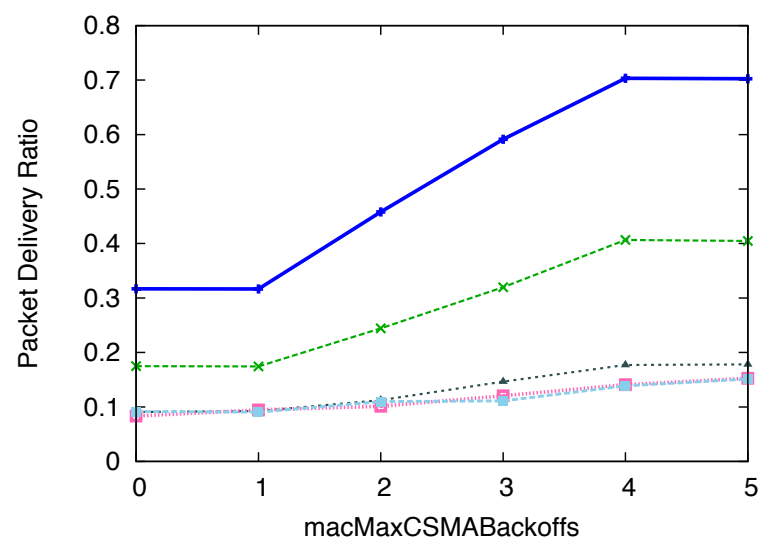

(b) Packet Delivery Ratio in function of macMaxCSMABackoffs

Fig. 2. Packet Delivery Ratio

assume that nodes always have a packet to send at the beginning of each superframe. Table I presents the simulation parameters.

\begin{tabular}{ll}
\multicolumn{1}{c}{ TABLE I. } & SIMULATION PARAMETERS \\
\hline Parameter & Description \\
\hline Simulated area & 100m x 100m \\
\hline Traffic & CBR $(1$ frame/node per superframe $)$ \\
\hline Simulation duration & $100000 \mathrm{~s}$ \\
\hline Propagation & Unit Disk Graph \\
\hline Duty cycle & $1 \%$ \\
\hline
\end{tabular}

\section{A. Impact of macMaxFrameRetries}

Figure 2(a) shows the impact of macMaxFrameRetries on the Packet Delivery Ratio in star topologies with the number of nodes from 4 to 64 . The parameter is in the interval between 0 and 7 with the default value of 4 , macMaxCSMABackoffs $=4$, $\operatorname{macMin} B E=3$, and $\operatorname{macMaxBE}=5$. We can observe that PDR remains constant for the values equal or greater than 2. Anastasi et al. [3] have already highlighted the fact that frames are very seldom dropped because of excessive number of retransmissions $(<2 \%$ when macMaxFrameRetries $=2)$.

\section{B. Impact of macMaxCSMABackoffs}

Figure 2(b) shows the impact of macMaxCSMABackoff on the Packet Delivery Ratio in a similar set up. The parameter is in the interval between 0 and 5 with the default value 


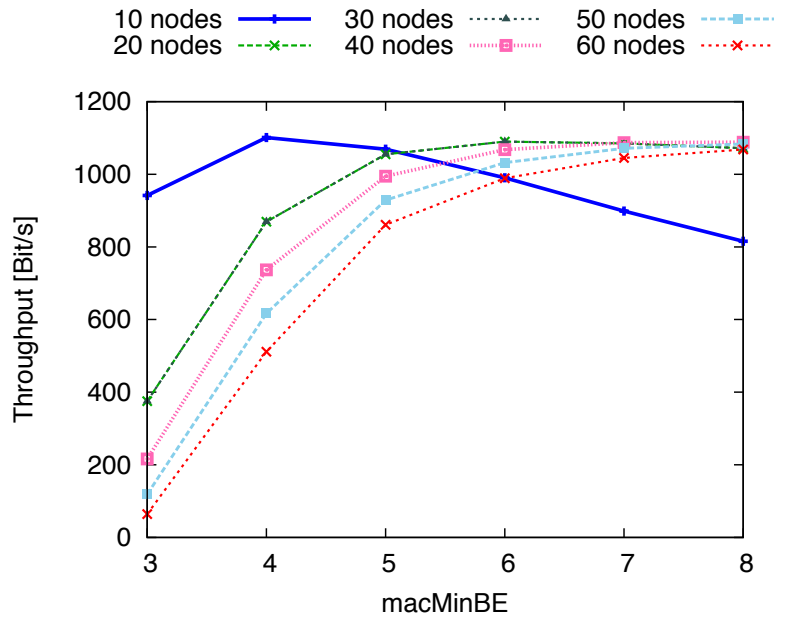

Fig. 3. Impact of macMinBE on the throughput

of 4 , macMaxFrameRetries $=4, \operatorname{macMinBE}=3$, and $\operatorname{macMaxBE}=5$. We first point out that greater values of macMaxCSMABackoff increase the PDR, because nodes have more opportunities of a successful transmission attempt. As at the beginning of an active period nodes try to access the channel to transmit frames buffered during the sleeping period, there is a high probability that nodes find the channel busy. Maintaining a large value of macMaxCSMABackoff contributes to better PDR.

We can observe that the two parameters have little influence on PDR for a large number of nodes, because in this case, contention results in many collisions and very low PDR.

\section{Influence of $B E$}

Finaly, we analyze the impact of the $B E$ parameter on the throughput. To investigate the contention behavior, we simulate a backlogged traffic - each node has always a frame to transmit in a set up with one coordinator and a variable number of associated nodes (nodes still operate with $1 \%$ duty cycle). Other parameters are set to their default values i.e macMaxFrameRetries $=3$ and macMaxCSMABackoff $=4$. Fig. 3 presents the throughput that strongly depends on the number of contending nodes with an optimal value of $B E$ for a given number of nodes.

We conclude from the above analysis that macMaxFrameRetries and macMaxCSMABackoffs should have the largest possible values (and not the default ones) and nodes need to adapt $B E$ to the current number of active contending nodes to increase the throughput. We propose a mechanism for $B E$ adaptation in the next section.

\section{Mechanism For AdAPting Contention Window}

We assume that the network operates with a low duty cycle set by a proper choice of the $B O$ and $S O$ parameters of the superframe. All contending nodes wake up at the instant of beacon transmission and try transmit their frames during the active period. Thus, even low traffic may lead to a high packet drop rate because of channel busy and high collision rate at the beginning of the active period if the number of nodes is important.
The objective of the mechanism is to adapt the contention window so that:

- the backoff is not too short to avoid collisions,

- the backoff is not too long, because in this case the channel is under-utilized resulting in a lower throughput.

To achieve this balance, the mechanism needs to find the $B E$ value that results in the optimal throughput by minimizing collisions, minimizing the number of idle slots between transmissions, and maximizing the successful transmissions.

\begin{tabular}{ll} 
& \multicolumn{1}{c}{ TABLE II. Model nOtATION } \\
\hline Variable & Description \\
\hline$B E$ & Backoff Exponent $\in[3,8]$ \\
\hline$B$ & $\begin{array}{l}\text { Random Backoff } \in\left[0, B_{\max }\right], B_{\max }=2^{B E}-1 \\
B E \text { takes the value of } \text { macMinBE parameter }\end{array}$ \\
\hline$P_{e}$ & $\begin{array}{l}\text { Transmission attempt probability, probability of } \\
\text { choosing a given slot for transmission }\end{array}$ \\
\hline$P_{c}$ & $\begin{array}{l}\text { Probability that several nodes transmit in the same } \\
\text { slot leading to a collision }\end{array}$ \\
\hline$P_{t}$ & Probability that a node transmits in a free slot \\
\hline$P_{i}$ & $\begin{array}{l}\text { Probability that no station transmits during a given } \\
\text { slot }\end{array}$ \\
\hline
\end{tabular}

\section{A. Modeling IEEE 802.15.4 CSMA/CA}

To derive the optimal adaptation of the contention window, we have analytically modeled the IEEE 802.15.4 CSMA/CA access method following the approaches proposed for IEEE 802.11 by Bianchi [8] and then Heusse et al. [4]. Table II summarizes the notation.

We start with the following assumptions to derive the model:

- backlogged traffic: nodes always have a packet to transmit,

- network topology: a star without hidden stations with a central coordinator acting as the PAN coordinator,

- a fixed contention window (fixed $B E, B$ chosen randomly in $\left[0,2^{B E}-1\right]$ ).

We show below that although the IEEE 802.15.4 CSMA/CA access method is very different from 802.11, we can express the probabilities defined in Table II as in the model of Bianchi [8] and Heusse et al. [4].

Transmission attempt probability $P_{e}$ that a node chooses a particular slot depends on the contention window [9]:

$$
P_{e}=\frac{2}{B_{\max }+1} \text {. }
$$

Transmission probability $P_{t}$ corresponds to the case in which a station transmit a frame without collision, i.e only one node has chosen to attempt a transmission in a given slot:

$$
P_{t}=N \times P_{e} \times\left(1-P_{e}\right)^{N-1},
$$

where $N$ is the number of contending nodes. A collision happens when two or more stations choose the same slot for a transmission attempt. Its probability is the following:

$$
P_{c}=1-P_{t}-P_{i}
$$




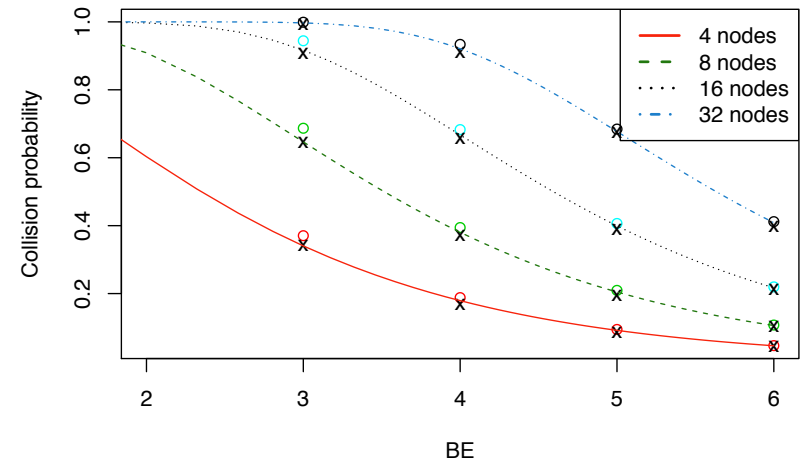

Fig. 4. Collision probability $P_{c}$ (WsNet - crosses, simulator in C - circles, analytical expressions - continuous lines).

A slot is considered idle if there is no transmission during this slot. The corresponding idle probability $P_{i}$ is as follows:

$$
P_{i}=\left(1-P_{e}\right)^{N}
$$

Let us denote by $n_{\mathrm{i}}$ the number of consecutive idle slots between two transmission attempts (transmission or collision). Its mean depends on the idle probability in the following way:

$$
\overline{n_{i}}=\frac{P_{i}}{1-P_{i}}
$$

We can observe that transmission attempt probability $P_{e}$ only depends on the size of the contention window $\left(B_{\max }=\right.$ $2^{B E}-1$ in IEEE 802.15.4 and $C W$ in IEEE 802.11). Other probabilities $P_{c}, P_{t}$, and $P_{i}$ only depend on $P_{e}$ and on the number of contending nodes. As all nodes make their choices independently, probability $P_{e}$ is independent of what happens in neighboring slots. Moreover, it does not change if some slots are occupied with a transmission. As slots are independent, freezing the countdown of a backoff when a transmission occurs on the channel as in IEEE 802.11 or continuing the countdown as in IEEE 802.15.4 result in the same probabilities.

\section{B. Model Validation}

To validate the surprising result that we can apply the 802.11 model to IEEE 802.15.4, we have compared the analytical results with simulations done using two simulators: WsNet [5] and a simple simulator in $\mathrm{C}$ that implements the IEEE 802.15.4 slotted MAC. We have simulated a star topology with an increasing number of nodes around the PAN coordinator from 4 to 32 nodes with various contention window sizes $\left(B_{\max }, B E \in[3,4,5,6]\right)$.

Figures 4 and 5 represent collision probability $P_{c}$ and $\overline{n_{i}}$, the average number of idle slots before a transmission in function of $B E$, respectively. The results obtained with WsNet, the simulator in $\mathrm{C}$, and the analytical expressions above are presented with crosses, circles, and continuous lines, respectively. We can observe fairly good fit between the model and the simulation results.

\section{ABE Mechanism - Adapting Contention Window}

Based on the model above (Eqs. 1-5), we can optimize the throughput:

$$
X\left(P_{e}\right)=\frac{P_{t} \times s_{d}}{P_{t} \times T_{t}+P_{c} \times T_{c}+P_{i} \times T_{s l o t}},
$$

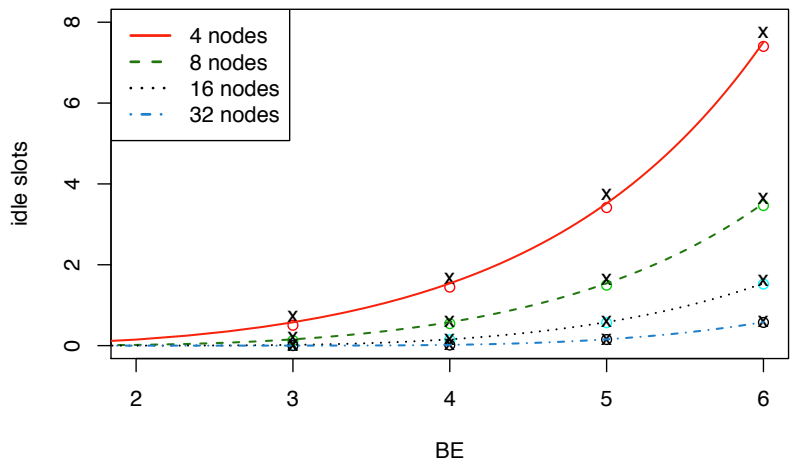

Fig. 5. $\overline{n_{i}}$, average number of idle slots before a transmission (WsNet crosses, simulator in $\mathrm{C}$ - circles, analytical expressions - continuous lines)

where $T_{t}$ represents the average transmission time, $T_{c}$ collision time, $T_{\text {slot }}$ time slot, and $s_{d}$ the average frame size, to find the optimal contention window [4]. We maximize the throughput by minimizing the following cost function:

$$
\operatorname{cost}\left(P_{e}\right)=\frac{\frac{T_{c}}{T_{s l o t}} \times P_{c}+P_{i}}{P_{t}}
$$

Setting the first derivative of the cost function to zero leads to:

$$
1-N P_{\mathrm{e}}^{\mathrm{opt}}=\eta\left(1-P_{\mathrm{e}}^{\mathrm{opt}}\right)^{N}
$$

where

$$
\eta=1-\frac{T_{s l o t}}{T_{c}}
$$

can be computed from the parameters of the MAC and PHY layers. By denoting

$$
\zeta=N P_{e}^{\mathrm{opt}},
$$

we obtain: $1-\zeta=\eta(1-\zeta / N)^{N}$. If we consider the limit for $N \rightarrow \infty$, it gives:

$$
1-\zeta=\eta e^{-\zeta}
$$

We can solve this equation numerically for a given value of $\eta$. Moreover, when $N \rightarrow \infty$, Eq. 4 yields

$$
P_{\mathrm{i}}^{\mathrm{opt}}=(1-\zeta / N)^{N} \rightarrow e^{-\zeta} \text {. }
$$

This means that for a given ratio $\frac{T_{\mathrm{c}}}{T_{\text {slot }}}$, the throughput is optimal for the probability of an idle slot $P_{\mathrm{i}}^{\text {opt }}$ that tends towards an easily computable constant. Similarly, Eq. 5 gives the optimal number of idle slots between two transmission attempts when $N \rightarrow \infty$ :

$$
\bar{n}_{\mathrm{i} \infty}^{\mathrm{opt}}=\frac{e^{-\zeta}}{1-e^{-\zeta}} .
$$

Its value for IEEE 802.15.4 is $\bar{n}_{\mathrm{i} \infty}^{\mathrm{opt}}=1.4366$ and from Eq. 5, we obtain:

$$
P_{\mathrm{i} \infty}^{o p t}=\frac{1.4366}{2.4366}
$$

We can build an adaptation mechanism on this result in a similar way to Idle Sense: nodes may observe the average number of idle slots between two transmission attempts to derive the level of contention on the channel.

If average number of idle slots is less than $\bar{n}_{\mathrm{i}}^{\text {opt }}$ for a given number of contending nodes $N$, the operating point is not optimal due to excessive collisions, nodes thus need to increase 
$B_{\max }$; conversely, if the observed value is greater than $n_{\mathrm{i}}^{\mathrm{opt}}$, the operating point is not optimal due to too much time spent waiting during idle slots, nodes thus need to decrease $B_{\max }$.

As for 802.11 wireless LANs, the adaptation method targets the value of $\bar{n}_{\mathrm{i} \infty}^{\mathrm{opt}}$ as the ideal operation point instead of $\bar{n}_{\mathrm{i}}^{\text {opt }}$ for any number of active stations $N$. This difference has little effect on performance and allows to stay on the safe side of the optimal operating point: we can observe in Figure 3 that the throughput drops much faster for a smaller $B E$ compared to a larger one. In the remainder of this section, we denote by $\bar{n}_{\mathrm{i}}^{\text {target }}=\bar{n}_{\mathrm{i} \infty}^{\mathrm{opt}}$ the target average number of idle slots that the adaptation algorithm will try to maintain.

However, the problem with this approach is energy consumption - if a node wants to observe idle slots, it needs to be awake and consume energy. When a node goes to sleep during the backoff, it does not have the information for adaptation. We propose to solve this problem by delegating tracking of idle slots to the coordinator that anyway needs to stay awake during the active period. It observes the number of idle slots during the current superframe to estimate its average number $\widehat{n}_{i}$ (and so $\widehat{P}_{i}$ from relation 5). From this value, it then derives the average number of contending stations $\widehat{N}$. Note that simple observation of transmissions on the channel does not allow to estimate the number of active stations, because the coordinator does not perceive all unsuccessful transmission attempts. From $\widehat{N}$ and $\bar{n}_{\mathrm{i}}^{\text {target }}$, the coordinator then finds the value of $B_{\max }$ for reaching $\bar{n}_{\mathrm{i}}^{\text {target }}$ during the next superframe and sends $B_{\max }$ to the associated nodes in the next beacon.

More specifically, the adaptation algorithm operates as follows. At superframe $k$, the coordinator knows:

- $\bar{n}_{\mathrm{i}}^{\text {target }}$ (and so $P_{i}^{\text {target }}$ from Eq. 5),

- $\quad$ current $B_{\max }^{k}$ (and so $P_{\mathrm{e}}^{k}$ from Eq. 1).

After observing the traffic during the active period of superframe $k$, the coordinator:

- $\quad$ first, estimates the average number of idle slots $\widehat{n}_{i}^{k}$ (and so $\widehat{P}_{i}^{k}$ from Eq. 5);

- then, it derives the average number of active nodes from Eq. 4:

$$
\widehat{N}^{k}=\frac{\log \left(\widehat{P}_{i}^{k}\right)}{\log \left(1-P_{e}^{k}\right)}
$$

- from which the transmission attempt probability for the next superframe is computed based on $P_{i}^{\text {target }}$ :

$$
P_{\mathrm{e}}^{k+1}=1-\left(P_{\mathrm{i}}^{\mathrm{target}}\right)^{1 / \widehat{N}}
$$

- that finally allows computing the contention window for the next superframe:

$$
B_{\max }^{k+1}=\frac{2}{P_{\mathrm{e}}^{k+1}}-1 .
$$

\section{Adaptation for Bursty Traffic}

The prediction of the target values for the next superframe based on the values observed in the current one is satisfactory if the traffic pattern changes slowly. However, if there is no traffic in the previous superframe, the coordinator sets the value of $B_{\max }^{k+1}$ to its minimum ( $\left.\operatorname{macMin} B E=3\right)$, which may result in a high collisions rate if nodes in the next superframe happen to have frames to send. In such situations with traffic concentrated on a subset of superframes, the coordinator will not be able to derive the correct estimation of MAC parameters for a future superframe based on a superframe with no traffic.

To handle such irregular or bursty traffic, we propose to set $B_{\max }^{k+1}$ to its maximum value $(\operatorname{macMin} B E=8)$ for the next superframe, if there is no traffic in the current one. This rule avoids collisions and dropping packets in a superframe with bursts of traffic. The coordinator also applies the same rule upon initialization.

\section{E. Adaptation of the Number of Channel Sensing}

If the packet generation rate is low (for example one frame per node), all nodes try to access the channel at the beginning of a superframe. The first nodes access the channel and can transmit their frames, while the others may drop their frames when channel is busy at the instants of channel sensing for macMaxCSMABackoff attempts. The coordinator is not aware that nodes may drop frames due to the initial period of high channel utilization, which leads to an incorrect estimation of $B_{\max }^{k+1}$.

There are two ways of avoiding frame drops in this situation. The first one is to extend the macMaxCSMABackoff parameter beyond its possible values defined in the standard, for instance up to 8 (Figure 2 suggests that large values contribute to better PDR). Other analyses already proposed such an adjustment [3]. The drawback of this approach is an increased energy consumption required for CCAs. The second way is to count the number of unsuccessful CCAs instead of the number of backoffs (each channel sensing consists of 2 CCAs). For instance, as macMaxCSMABackoff $=4$ may result in 8 CCAs in the worst case, we will limit the number of CCAs to 8 . We have used the second way in our evaluation.

\begin{tabular}{ll}
\multicolumn{1}{c}{ TABLE III. } & Simulation Parameters \\
\hline Parameter & Value \\
\hline Simulated area & $100 \mathrm{~m} \mathrm{x} \mathrm{100m}$ \\
\hline Saturated Traffic & always packet to send \\
\hline non Saturated Traffic & VBR or Poisson \\
\hline Simulation duration & $100000 \mathrm{~s}$ \\
\hline Number of nodes & up to 32 \\
\hline Propagation & Unit Disk Graph \\
\hline Duty cycle & $1 \%$ \\
\hline Packet size & 30 bytes \\
\hline
\end{tabular}

\section{PERFormance EVAluation}

We have evaluated $\mathrm{ABE}$ and compared it with the IEEE 802.15.4 standard configured with static $B E$ values. As previously, we have used WsNet [5] with parameters given in Table III. We have first simulated a star network with an increasing number of nodes from 4 to 32 . The simulation results are the throughput of received data at the coordinator and the end-to-end delay (time between packet generation and reception by the coordinator) under our mechanism and the standard IEEE 802.15.4 CSMA/CA with $\operatorname{macMinBE}=3 \ldots 8$.

Figure 6 shows the throughput under saturated traffic (nodes have always a frame to send). We can observe that $\mathrm{ABE}$ provides optimum throughput regardless of the number 


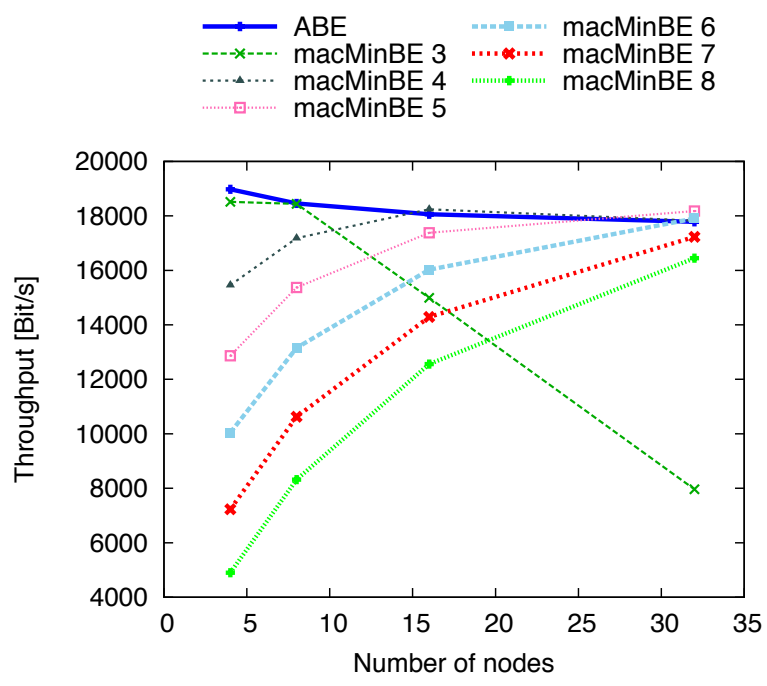

Fig. 6. Throughput under saturated traffic
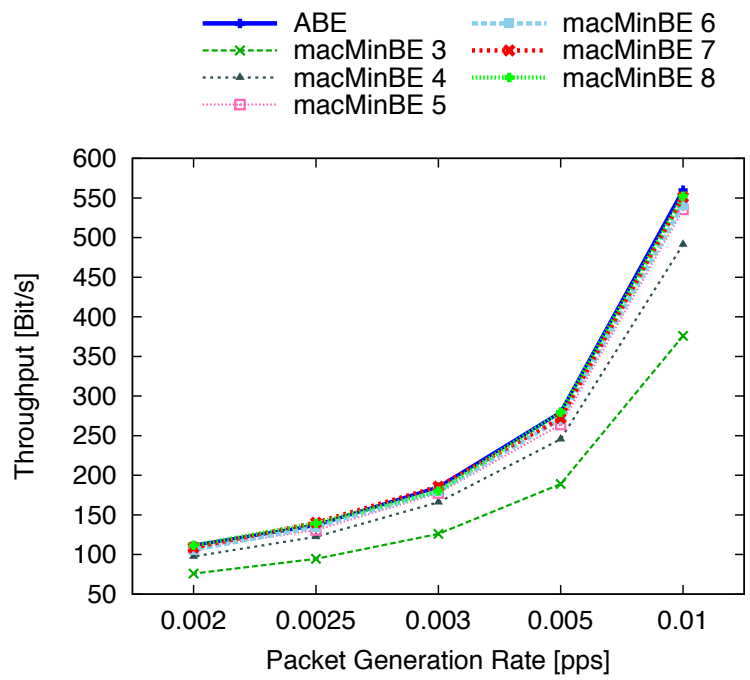

Fig. 7. Throughput under periodic non-uniform traffic

of nodes - the coordinator always finds the value of the contention window that optimizes the throughput. The standard CSMA/CA (macMinBe $=3$ ) presents good performance for a small number of nodes, but for a larger number of nodes, ABE results in substantial gains.

We have also considered other traffic conditions:

- Periodic non-uniform traffic (VBR): each node periodically generates a random number of packets under the constraint that the total number of generated packets is constant. This kind of traffic is extremely bursty and represents well the load put on a coordinator in a multi-hop cluster-tree topology where each coordinator receives traffic proportionally to the number of nodes in its sub-tree.

\section{- Poisson traffic of variable intensity.}

Figure 7 shows the throughput under periodic non-uniform traffic that stresses the proposed mechanism with the traffic pattern alternating superframes with and without traffic for a star network with 15 nodes. ABE manages well to maximize throughput for any traffic intensity. We have also tested the methods with a CBR traffic that leads to the same results, so we decided to not present them here.

Figures 8 and 9 present the throughput, the packet delivery ratio, and the delay under Poisson traffic for a star network with 5 and 20 nodes, respectively. ABE steadily maintains the throughput close to the optimal value for any level of contention. Its operation results in the best packet delivery ratio along with good delays - shorter delays are only obtained by the standard 802.15.4 method with the parameters leading to a lower packet delivery ratio. Overall, the proposed method provides very good performance over a wide range of the network size and traffic intensity, which is not possible to achieve in the standard 802.15.4 network with constant parameters.

\section{RELATED WORK}

Many authors studied the performance of IEEE 802.15.4. Misic et al. modeled the beacon-enabled mode with a discrete time Markov chain [10]. They measured the impact of the service time and queue length distributions on the MAC performance to conclude that the choice of the frame length, traffic intensity, and the size of the network are important to maximize the throughput. Park et al. adopted a similar approach to measure the performance of the standard IEEE 802.15.4 in terms of reliability, energy consumption, and delays [11]. They noticed that large values of macMinBE, macMaxFrameRetries, and macmaxCSMABackoff improve performance. Koubaa et al. [12] studied the impact of different factors on performance through simulation. The simulation demonstrated that the value of macMinBE has a significant impact on the performance in terms of packet loss and latency. The authors also concluded that the default parameters of the IEEE 802.15.4 CSMA/CA are not suitable for all network conditions. Similarly, Anastasi et al. [3] concluded that the default parameters of the CSMA/CA lead to poor performance, because the value of macMinBE does not allow efficient medium access even for small networks.

Experimentally, Liu et al. [13] reported a poor delivery ratio in multihop topologies (less than $10 \%$ of packets are received by the sink for a tree depth greater than 5 hops). They also noticed that the throughput quickly degrades with the number of nodes even in star topologies. Lee et al. [14] also demonstrated performance degradation when the number of contending nodes increases. Park et al. proposed an analytical model based on Markov chains to find the optimal values of IEEE 802.15.4 parameters [15]. The approach requires a continuous estimation of the busy channel probability, which is difficult to apply in sensor networks with sleeping nodes. Rao et al. [16] proposed to tune dynamically macMinBE according to the following method. The coordinator observes the channel to identify active nodes that have sent frames during a superframe. It then increments $\operatorname{macMinBE}$ for the active nodes, decrements it for all others, and piggybacks these values onto beacons. The coordinator adapts macMinBE every 3 superframes. The adaptation takes a longer time compared to our approach and it is inefficient for varying traffic patterns that may change during the observation phase. The approach also suffers from the overhead of sending the addresses of all nodes to notify them which value of macMinBE to use. Anastasi et al. [17] proposed an adaptive protocol in which each node finds the best value for its IEEE 802.15.4 parameters. 

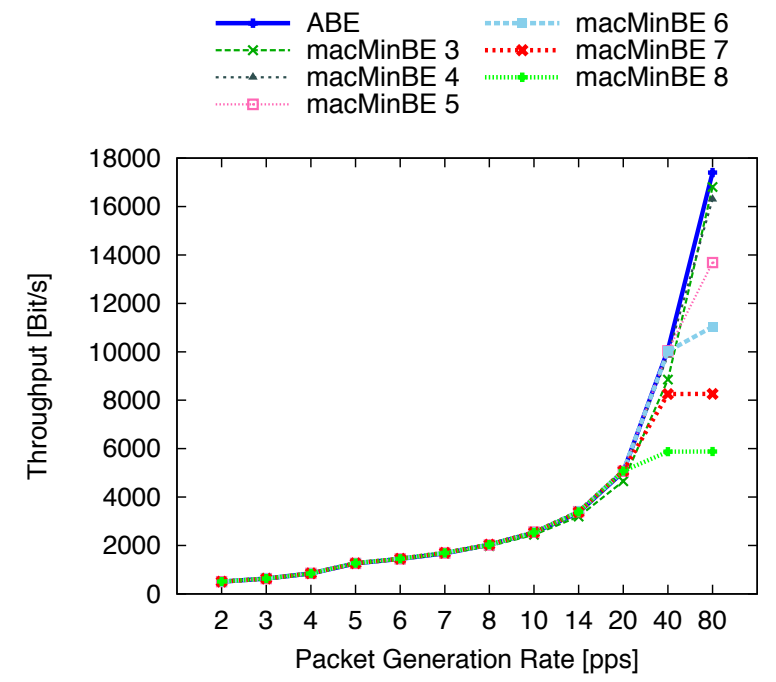

(a) Throughput (Bit/s)

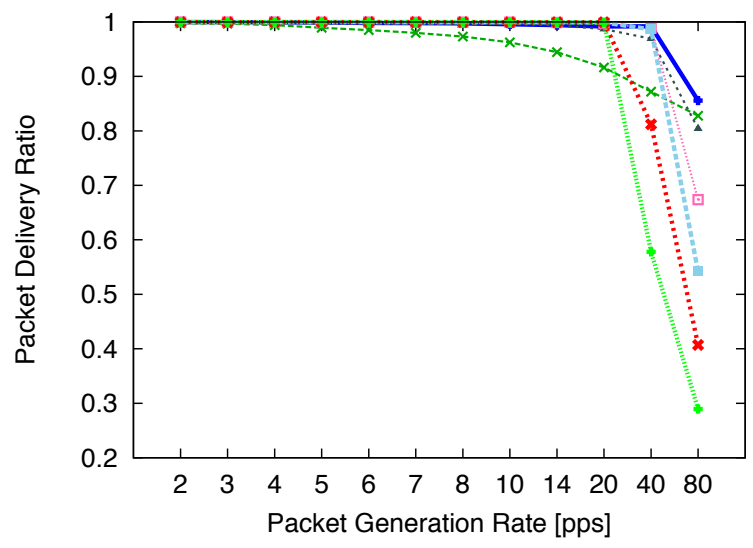

(b) Packet Delivery Ratio

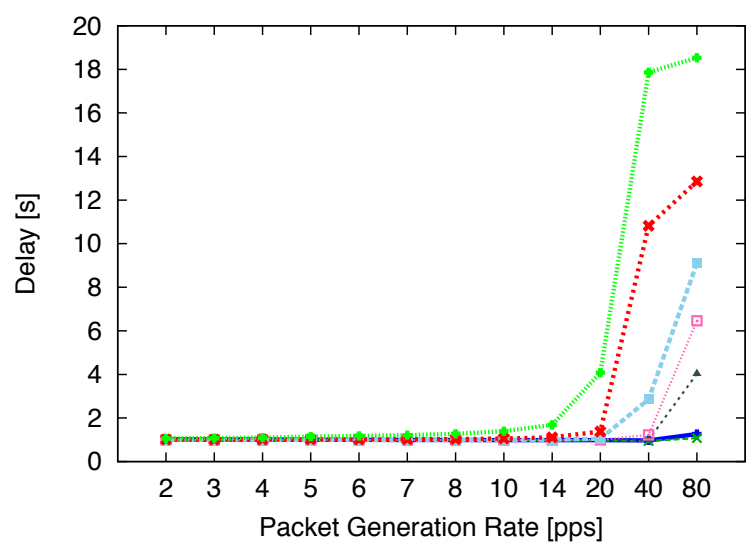

(c) Delay (s)

Fig. 8. Poisson traffic with 5 nodes

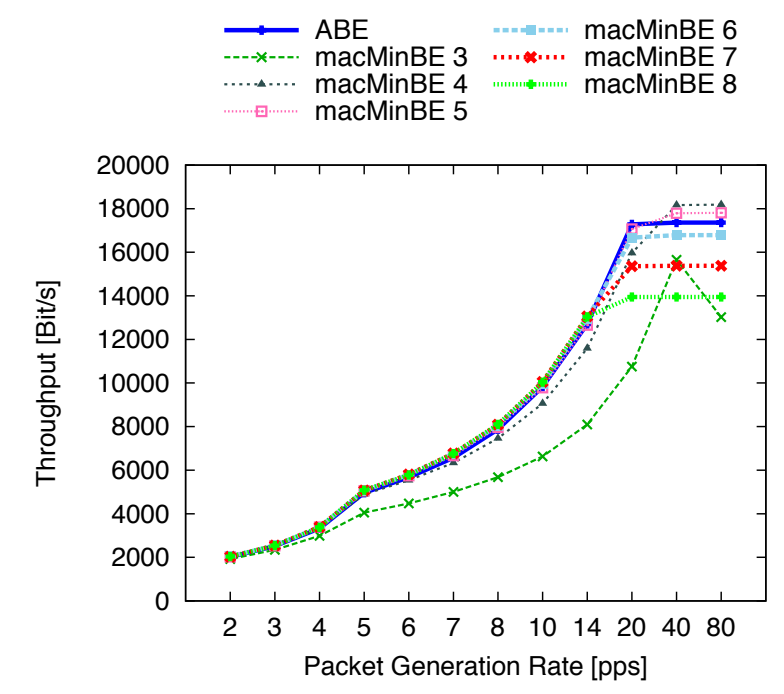

(a) Throughput (Bit/s)

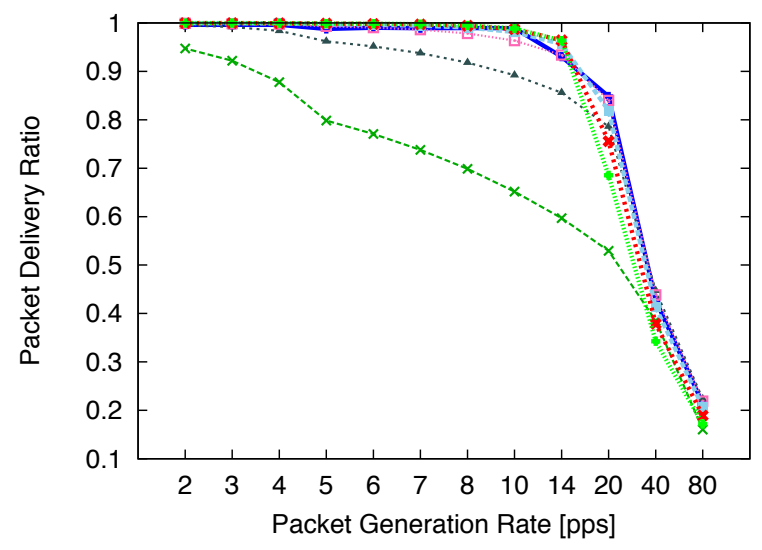

(b) Packet Delivery Ratio

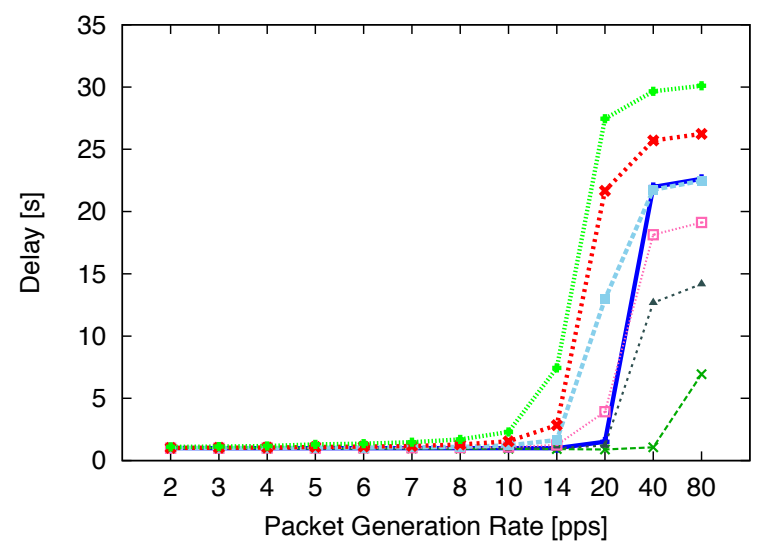

(c) Delay (s)

Fig. 9. Poisson traffic with 20 nodes 
The nodes continuously adapt macMinBE and macMAXCSMABackoff values to match the packet delivery ratio imposed by the application layer. Jamieson et al. [18] proposed Sift, a MAC protocol for wireless sensor networks. The protocol reduces the collision rate by using a truncated geometricallyincreasing probability distribution for picking a contention slot. The distribution approximates the optimal one that maximizes the probability of a successful transmission. Sift requires that nodes are synchronized and awake at the beginning of the contention period.

\section{CONCLUSION AND PERSPECTIVES}

In this paper, we have addressed the problem of optimizing the operation of IEEE 802.15.4 networks from a different point of view than existing approaches. We assume an energyefficient operation with low-duty cycles set up by a suitable combination of the superframe parameters $S O$ and $B O$. To optimize the performance of the access method during active periods, we propose to apply the approach based on Idle Sense in 802.11 networks that finds the right balance between the time spent in backoffs and collisions to maximize throughput. Even if the access methods of IEEE 802.15.4 and 802.11 are radically different, we can model them in a similar way in terms of transmission attempt, idle, and collision probabilities.

We have validated the model of IEEE 802.15.4 and used it to derive $\mathrm{ABE}$, an adaptation mechanism that varies the contention window in function of active nodes and changing traffic patterns. The proposed $\mathrm{ABE}$ method fundamentally relies on the special role played by a coordinator that observes the channel, derives its load, and distributes the value of the contention window to the associated devices. Moreover, we have designed $\mathrm{ABE}$ with bursty traffic conditions in mind, as they are inherent to the considered networks. Our simulation comparisons with the standard IEEE 802.15.4 show that the proposed method provides very good performance over a wide range of the network size and traffic intensity compared to the standard 802.15.4 network with constant parameters.

We plan to validate the proposed scheme on an experimental testbed to evaluate the impact of factors that appear in real radio environments. We also want to take into account phenomena that may appear in multi-hop topologies such as beacon collisions. We also plan consider managing differentiated access priorities that could reflect the depth of the subtree that each coordinator serves.

\section{ACKNOWLEDGEMENT}

dans, and the European Commission FP7 project CALIPSO under contract 288879.

\section{REFERENCES}

[1] IEEE 802.15.4, “WPAN task group 4," http://www.ieee802.org/15/pub/ TG4.html, 2006

[2] Y.-K. Huang, A.-C. Pang, and H.-N. Hung, "A Comprehensive Analysis of Low-Power Operation for Beacon-Enabled IEEE 802.15.4 Wireless Networks," IEEE Transactions on Wireless Communications, vol. 8 , no. 11 , november 2009.
[3] G. Anastasi, M. Conti, and M. Di Francesco, "The MAC Unreliability Problem in IEEE 802.15.4 Wireless Sensor Networks," in International Conference on Modeling, Analysis and Simulation of Wireless and Mobile Systems (MSWiM). Tenerife, Spain: ACM, October 2009.

[4] M. Heusse, F. Rousseau, R. Guillier, and A. Duda, "Idle Sense: An Optimal Access Method for High Throughput and Fairness in Rate Diverse Wireless LANs," in Proceedings of the 2005 conference on Applications, technologies, architectures, and protocols for computer communications, ser. SIGCOMM '05. New York, USA: ACM, 2005.

[5] A. Fraboulet, G. Chelius, and E. Fleury, "Worldsens: Development and Prototyping Tools for Application Specific Wireless Sensors Networks," in IPSN'07 Track on Sensor Platforms, Tools and Design Methods (SPOTS). Cambridge, Massachusetts, USA.: ACM, April 2007.

[6] N. Abdeddaim and F. Theoleyre, "Implementation of a WSNet Module to Simulate the IEEE 802.15.4 Beacon-Enabled Mode in Multihop Topologies," HAL, Research Report 00590853 http://hal. archives-ouvertes.fr/hal-00590853, May 2011.

[7] H. Jeon and Y. Kim, "BOP (Beacon-Only Period) and Beacon Scheduling for MEU (Mesh-Enabled USN) Devices," in Proceedings of ICACT, February 2007.

[8] G. Bianchi, "Performance Analysis of the IEEE 802.11 Distributed Coordination Function," IEEE Journal on Selected Areas in Communications, vol. 18 , no. 3 , pp. $535-547$, mar 2000.

[9] G. Bianchi, L. Fratta, and M. Oliveri, "Performance Evaluation and Enhancement of the CSMA/CA MAC Protocol for 802.11 Wireless LANs," in Proc. of PIMRC'96, vol. 2, Oct 1996, pp. 392 -396.

[10] J. Misic, S. Shafi, and V. B. Misic, "The Impact of MAC Parameters on The Performance of 802.15.4 PAN," Ad Hoc Networks, vol. 3, no. 5, pp. $509-528,2005$.

[11] P. Park, P. Di Marco, P. Soldati, C. Fischione, and K. Johansson, "A Generalized Markov Chain Model for Effective Analysis of Slotted IEEE 802.15.4," in International Conference on Mobile Adhoc and Sensor Systems (MASS). IEEE, oct. 2009, pp. $130-139$.

[12] A. Koubaa, M. Alves, and E. Tovar, "A Comprehensive Simulation Study of Slotted CSMA/CA for IEEE 802.15.4 Wireless Sensor Networks," in Factory Communication Systems, 2006 IEEE International Workshop on, 2006.

[13] X. Liu, C. Leckie, and S. Saleem, "Performance Evaluation of a Converge-Cast Protocol for IEEE 802.15.4 Tree-Based Networks," in International Conference on Intelligent Sensors, Sensor Networks and Information Processing ISSNIP. IEEE, dec. 2010, pp. 73 -78.

[14] J.-S. Lee, "An Experiment on Performance Study of IEEE 802.15.4 Wireless Networks," in Emerging Technologies and Factory Automation, 2005. ETFA 2005. 10th IEEE Conference on, vol. 2, sept. 2005

[15] P. Park, C. Fischione, and K. H. Johansson, "Adaptive IEEE 802.15.4 Protocol for Energy Efficient, Reliable and Timely Communications," in International Conference on Information Processing in Sensor Networks (IPSN). Stockholm, Sweden: ACM/IEEE, 2010, pp. 327-338.

[16] V. Rao and D. Marandin, "Adaptive Backoff Exponent Algorithm for Zigbee (IEEE 802.15.4)," in Next Generation Teletraffic and Wired/Wireless Advanced Networking, ser. Lecture Notes in Computer Science, Y. Koucheryavy, J. Harju, and V. Iversen, Eds. Springer Berlin / Heidelberg, 2006, vol. 4003, pp. 501-516, 10.1007/11759355-46.

[17] M. D. Francesco, G. Anastasi, M. Conti, S. K. Das, and V. Neri, "Reliability and Energy-Efficiency inIEEE 802.15.4/ZigBee Sensor Networks: An Adaptive and Cross-Layer Approach," IEEE Journal on Selected Areas in Communications, vol. 29, no. 8, pp. 1508-1524, 2011.

[18] K. Jamieson, H. Balakrishnan, and Y. C. Tay, "Sift: a MAC Protocol for Event-Driven Wireless Sensor Networks," in Proceedings of the European Conference on Wireless Sensor Networks (EWSN), Zurich, Switzerland, Feb. 2006. 